(2) Open Access Full Text Article

\title{
Ferumoxytol versus placebo in iron deficiency anemia: efficacy, safety, and quality of life in patients with gastrointestinal disorders
}

This article was published in the following Dove Press journal:

Clinical and Experimental Gastroenterology

II July 2016

Number of times this article has been viewed

\author{
David C Ford' \\ Naomi $\vee$ Dahl $^{2}$ \\ William E Strauss ${ }^{2}$ \\ Charles F Barish ${ }^{3}$ \\ David J Hetzel ${ }^{4}$ \\ Kristine Bernard ${ }^{2}$ \\ Zhu $\mathrm{Li}^{2}$ \\ Lee F Allen ${ }^{2}$
}

'Toronto Digestive Disease Associates, Inc, Vaughan Endoscopy Clinic, Vaughan, ON, Canada; ${ }^{2}$ AMAG Pharmaceuticals, Inc, Waltham, MA, ${ }^{3}$ Wake Gastroenterology, Wake Research Associates, Raleigh, NC, USA; ${ }^{4}$ Department of Gastroenterology, Royal Adelaide Hospital, Adelaide, SA, Australia
Correspondence: William E Strauss Medical Affairs, AMAG Pharmaceuticals, Inc, I I00 Winter Street, Waltham, MA 0245I, USA

Tel + I 6174987789

Fax +I 6179022420

Email wstrauss@amagpharma.com
Introduction: Iron deficiency anemia (IDA) is common in patients with gastrointestinal (GI) disorders and can adversely affect quality of life. Oral iron is poorly tolerated in many patients with GI disorders. Ferumoxytol is approved for the intravenous treatment of IDA in patients with chronic kidney disease. This study aimed to evaluate the efficacy and safety of ferumoxytol in patients with IDA and concomitant GI disorders.

Patients and methods: This analysis included 231 patients with IDA and GI disorders from a Phase III, randomized, double-blind, placebo-controlled trial evaluating ferumoxytol $(510 \mathrm{mg} \times 2)$ versus placebo in patients who had failed or were intolerant of oral iron therapy. The primary study end point was the proportion of patients achieving a $\geq 20 \mathrm{~g} / \mathrm{L}$ increase in hemoglobin $(\mathrm{Hgb})$ from baseline to Week 5. Other end points included mean change in Hgb, proportion of patients achieving $\mathrm{Hgb} \geq 120 \mathrm{~g} / \mathrm{L}$, mean change in transferrin saturation, and patient-reported outcomes (PROs).

Results: Significantly more patients with IDA receiving ferumoxytol achieved a $\geq 20 \mathrm{~g} / \mathrm{L}$ increase in $\mathrm{Hgb}$ versus placebo $(82.1 \%$ vs $1.7 \%$, respectively; $P<0.001)$. Mean increase in $\mathrm{Hgb}(28.0 \mathrm{~g} / \mathrm{L}$ vs $-1.0 \mathrm{~g} / \mathrm{L}$, respectively; $P<0.001)$ significantly favored ferumoxytol treatment. Ferumoxytol-treated patients demonstrated significantly greater improvements than placebo-treated patients relative to their very poor baseline PRO scores posttreatment, including improvements in the Functional Assessment of Chronic Illness Therapy-Fatigue questionnaire and various domains of the 36-Item Short-Form Health Survey. Ferumoxytol-treated patients had a low rate of adverse events.

Conclusion: In this study, ferumoxytol was shown to be an efficacious and generally welltolerated treatment option for patients with IDA and underlying GI disorders who were unable to use or had a history of unsatisfactory oral iron therapy.

Keywords: hemoglobin, efficacy, inflammatory bowel disease, quality of life, patient-reported outcomes, intravenous iron

\section{Introduction}

Iron deficiency anemia (IDA) is common in patients with gastrointestinal (GI) disease. The anemia may be the result of chronic blood loss, malnutrition, or malabsorption of iron, and it often coexists with impaired utilization of endogenous iron in patients with chronic inflammatory disease. Chronic occult GI bleeding is a common cause of IDA, particularly among men and postmenopausal women. ${ }^{1-3}$ It is estimated that between $43 \%$ and $86 \%$ of patients with IDA have a GI pathology. ${ }^{4}$ GI-related causes of IDA include inflammatory bowel disease (IBD), colon cancer, or polyps, ${ }^{4}$ in addition to mucosal damage from use of aspirin or nonsteroidal anti-inflammatory drugs. 
Patients with malabsorption syndromes (eg, celiac disease) and vascular abnormalities such as Osler-Weber-Rendu syndrome, or those who have undergone postgastric bypass or gastrectomy, are also at increased risk of developing IDA. IDA can substantially impair patients' health-related quality of life (HRQoL), as assessed by patient-reported outcome (PRO) questionnaires, and their ability to work, with these patients often experiencing fatigue or impaired physical and/ or cognitive functioning. ${ }^{5-7}$

Diagnosis and correction of the underlying cause of IDA and repletion of iron stores are fundamental approaches to the management and treatment of these patients. ${ }^{4}$ While oral iron supplementation is recommended as a simple and safe first-line treatment for IDA, many patients, particularly those with comorbid GI disease, cannot take oral iron, do not tolerate it, or do not respond with adequate replenishment of iron stores. GI side effects including nausea, vomiting, and abdominal pain, or discomfort may be intolerable in these patients $^{4,8}$ and may lead to an exacerbation of existing GI symptoms. ${ }^{9}$ In addition, oral iron supplementation typically takes at least 2-3 weeks to increase the hemoglobin $(\mathrm{Hgb})$ concentrations and up to 2 months to achieve normal values; at least 6 months of compliant daily treatments are needed to replenish iron stores completely. ${ }^{4}$ Because patients are frequently noncompliant with oral iron treatment and discontinue it, many patients with IDA may live with chronic anemia and its related adverse effects on their quality of life. Therefore, the administration of intravenous (IV) iron may be the preferred treatment for IDA in many patients with GI disorders. ${ }^{4,9,10}$ Recent evidence-based international guidelines for the management of anemia in patients with IBD recommend IV iron as more effective and better tolerated than oral iron. ${ }^{11}$ The efficacy of IV iron supplementation in the treatment of IDA has been studied in patients with a variety of underlying conditions, including chronic kidney disease (CKD), abnormal uterine bleeding, pregnancy, postpartum anemia, cancer, and GI disorders, including IBD and GI blood loss. However, few placebo-controlled studies have been conducted specifically in patients intolerant to oral iron.

In the USA, both iron dextran and ferric carboxymaltose have a broad indication for the treatment of IDA in patients who do not respond to oral iron. The other available IV iron products, such as iron sucrose, ferric gluconate, and ferumoxytol, are approved only for the treatment of IDA in patients with CKD.

Ferumoxytol is a superparamagnetic iron oxide in a nondextran, semisynthetic, carbohydrate shell composed of polyglucose sorbitol carboxymethyl ether (PSC). The PSC coating was specifically engineered to help isolate the bioactive iron cores from plasma components until the iron-PSC complex enters the reticuloendothelial system macrophages, to reduce potential toxicities. The formulation is isotonic with a neutral $\mathrm{pH}(6.0-8.0)$ and very low free iron. ${ }^{12,13}$ Ferumoxytol was approved for use by the US Food and Drug Administration for the treatment of IDA in 2009 in adults with CKD. Supplemental to the original drug application, ferumoxytol is also being investigated in patients for the broad indication of the treatment of IDA in those patients who have failed treatment with or who are intolerant of oral iron therapy.

A Phase III study comparing ferumoxytol with placebo was completed as part of an overall program evaluating ferumoxytol for the treatment of IDA in patients with a history of unsatisfactory oral iron therapy or in whom oral iron could not be used (ClinicalTrials.gov identifier: NCT01114139). ${ }^{14}$ In this study, the primary end point of a Hgb increase of $\geq 20 \mathrm{~g} / \mathrm{L}$ at Week 5 was achieved in a statistically significantly greater proportion of ferumoxytol-treated patients compared with those receiving placebo $(81.1 \%$ vs $5.5 \%$, respectively; $P<0.001)$. Patients in the ferumoxytol treatment group also experienced a statistically significantly greater mean change in $\mathrm{Hgb}$ from baseline to Week 5 (the alternative primary study end point) than those in the placebo group $(27.0 \mathrm{~g} / \mathrm{L}$ vs $1.0 \mathrm{~g} / \mathrm{L}$, respectively; $P<0.001$ ). In addition, ferumoxytol treatment was associated with improvements in several quality of life scales and a low overall rate of adverse events (AEs). Therefore, we hypothesize that patients with IDA and concomitant GI disorders who had been unsuccessfully treated with or could not tolerate oral iron would tolerate ferumoxytol treatment as satisfactorily as the overall population of patients with IDA and show clinically important improvements in PROs and the correction of Hgb levels. Here, we report the efficacy and safety results from a prespecified secondary analysis of these Phase III clinical trial data that include the subgroup of patients with IDA and underlying GI disorders (IBD, malabsorption, and other causes of chronic GI blood loss).

\section{Patients and methods}

\section{Study design and medication}

The primary study was a Phase III, randomized, double-blind, placebo-controlled, multicenter trial conducted at 182 sites in the USA, Canada, Poland, Hungary, Latvia, and India from June 2010 through February 2012. ${ }^{14}$ Following a 2-week screening period, patients with IDA were randomized 3:1 to receive a $510 \mathrm{mg}$ dose of ferumoxytol (AMAG Pharmaceuticals, Waltham, MA, USA) (volume: $17 \mathrm{~mL}$ ) or normal saline 
placebo at the baseline visit (Day 1), followed by a second dose 2-8 days later (Week 1). Patients were observed weekly until the end of the 5-week treatment period (Weeks 2-5). The study was conducted with adherence to and compliance with the Declaration of Helsinki and Good Clinical Practice Guidelines. The study protocol was reviewed and approved by an institutional review board (IRB) at each study center, and patients provided written informed consent prior to study start. As this was an analysis of a prespecified subgroup of the overall study described that had IRB approval, this subsequent analysis did not require IRB approval.

\section{Patient population}

A full description of the inclusion and exclusion criteria has been published previously. ${ }^{14}$ Briefly, eligible patients were males and nonpregnant, nonbreastfeeding females $\geq 18$ years of age with a serum $\mathrm{Hgb}$ level between $70 \mathrm{~g} / \mathrm{L}$ and $<100 \mathrm{~g} / \mathrm{L}$ and a transferrin saturation (TSAT) value $<20 \%$. Eligible patients had either failed oral iron therapy or were intolerant to oral iron. Patients were excluded if they had a history of allergy to IV iron or serum ferritin $>1,348 \mathrm{pmol} / \mathrm{L}$, anemia due to other known causes excluding iron deficiency, hematologic malignancy, or were on dialysis or had an estimated glomerular filtration rate $<30 \mathrm{~mL} / \mathrm{min} / 1.73 \mathrm{~m}^{2}$. Those who received parenteral iron therapy prior to screening, another investigational agent within 4 weeks, or oral iron or a blood transfusion within 2 weeks were also excluded.

\section{Study assessments and end points}

Blood samples were collected at screening, baseline, and at weekly visits (Weeks 2-5) to assess efficacy ( $\mathrm{Hgb}$, TSAT, and other iron measures). Three PRO questionnaires were administered at baseline (Functional Assessment of Chronic Illness Therapy-Fatigue [FACIT-Fatigue], ${ }^{15}$ the 36-Item Short-Form Health Survey [SF-36], ${ }^{16}$ and the Linear Analog Scale Assessment [LASA]). ${ }^{17}$ The FACIT-Fatigue is a 13-item patient-reported assessment of fatigue that is scored on a $0-4$ response scale ranging from "Not at all" to "Very much so". To score the FACIT-Fatigue, all items are summed to create a single fatigue score with a range from 0 to 52 , with higher scores representing better functioning or less fatigue. The SF-36 is a validated generic HRQoL instrument that includes 36 items assessing patient health across eight domains: bodily pain, general health perceptions, mental health, physical functioning, role limitations due to emotional health problems, role limitations due to physical health problems, social functioning, and vitality. From the individual subscales, two component summary scores are generated: the physical component summary (PCS) and the mental component summary (MCS). The scores for each subscale are converted to norm-based $Z$-scores (based on 1998 US general population), with a mean of 50 and a standard deviation (SD) of ten. A score of 100 represents the best health. The LASA consists of three visual analog scales, one for each of the following domains: energy level, activities of daily living, and overall quality of life. Each visual analog scale consists of a $100 \mathrm{~mm}$ line with a left anchor representing the worst possible score (0) and a right anchor representing the best possible score (100), with higher scores indicative of better functioning or HRQoL.

The FACIT-Fatigue was administered weekly during Weeks 1-5, while the SF-36 and LASA were administered at Weeks 3 and 5 . A clinically meaningful difference or minimal important difference (MID) in score has been reported from other studies as 3.0 for the FACIT-Fatigue, ${ }^{18,19} 5.0$ for SF-36 domains, ${ }^{20}$ and $9.61,8.74$, and 9.81 for the LASA energy, activities of daily living, and quality of life domains, respectively. ${ }^{17}$ Patients in this study were assessed for AEs throughout the study and monitored for postadministration AEs, including hypersensitivity and hypotension, for a 60-minute period after each study drug administration. The severity of AEs and relationship to the study drug were assessed by study investigators. Additional safety assessments included vital signs, routine laboratory tests, concomitant medications, and physical examinations.

Efficacy end points of this GI subgroup analysis were the same as the overall original Phase III study. ${ }^{14}$ Two prespecified, separate primary efficacy analyses were planned to address the requests of various health authorities. For one analysis, the primary efficacy end point was the proportion of patients achieving a $\geq 20 \mathrm{~g} / \mathrm{L}$ increase in $\mathrm{Hgb}$ at any time from baseline to Week 5. For the other preplanned analyses, the alternative primary end point was the mean change in $\mathrm{Hgb}$ from baseline to Week 5. Secondary end points included the proportion of patients achieving a $\mathrm{Hgb} \geq 120 \mathrm{~g} / \mathrm{L}$ at any time from baseline to Week 5 and the mean change in TSAT and FACIT-Fatigue from baseline to Week 5. Exploratory end points included PRO assessments that evaluated the mean changes in SF-36 and LASA domains from baseline to Week 5.

Safety assessments included the incidence of treatmentemergent AEs (TEAEs), serious AEs (SAEs), severe AEs, any AEs leading to withdrawal of study treatment, AEs leading to study withdrawal, AEs leading to death, and two composite end points that were agreed upon a priori with regulatory agencies: AEs of special interest (AESIs; predefined as 
moderate-to-severe hypotension occurring on the day of dosing, and moderate-to-severe hypersensitivity reactions occurring within 48 hours postdose) and a composite cardiovascular end point (predefined as nonfatal myocardial infarction, heart failure, moderate-to-severe hypertension, and hospitalization due to any cardiovascular event).

\section{Statistical methods}

The intent-to-treat population and the safety population were identical and consisted of all randomized patients with exposure to study drug (ferumoxytol or placebo). Summary statistics (number of observations, mean, SD, and so on) were used to describe all continuous end points. Categorical end points were described by the frequency and percentage of patients. For the primary end point and the proportion of patients with $\mathrm{Hgb} \geq 120 \mathrm{~g} / \mathrm{L}$ at any time from baseline to Week 5, $P$-values were calculated by the Cochran-MantelHaenszel test. $P$-values for the alternative primary end point and all other continuous efficacy end points were calculated using an analysis of covariance model, with baseline $\mathrm{Hgb}$ level included as a covariate. No adjustments were made for multiple comparisons (ie, potential for type I error). Statistical significance (and superiority) for all $P$-values was defined as $P \leq 0.05$.

\section{Results}

The intent-to-treat subgroup of patients with IDA and an underlying GI disorder consisted of a total of 231 of the
$808(28.5 \%)$ randomized patients from the primary study, with 173 patients randomized to receive ferumoxytol and 58 patients randomized to receive placebo (Figure 1). Underlying GI conditions included gastric bypass (38.1\%), gastroesophageal reflux disease (35.1\%), IBD (21.2\%), GI bleeding-related condition (16.0\%), gastritis (15.6\%), ulcerrelated condition $(10.8 \%)$, and others $(6.9 \%)$, with some patients having more than one condition. Eight patients with IBD (ferumoxytol, $n=6$; placebo, $n=2$ ) received therapy with an anti tumor necrosis factor alpha agent. Most baseline demographics were evenly distributed between the ferumoxytol and the placebo treatment groups of the GI subgroup (Table 1). The mean baseline Hgb was similar in the two treatment groups: $89 \mathrm{~g} / \mathrm{L}$ for the ferumoxytol treatment group, compared with $87 \mathrm{~g} / \mathrm{L}$ for the placebo treatment group.

\section{Efficacy analyses}

The proportion of patients with an increase in Hgb of $\geq 20 \mathrm{~g} / \mathrm{L}$ from baseline to Week 5 (primary study end point) was $82.1 \%(n=142 / 173)$ for ferumoxytol-treated patients in the GI disorders subgroup compared with only $1.7 \%(n=1 / 58)$ of patients in the placebo treatment group (treatment difference: $80.4 \% ; P<0.001$ ) (Table 2). These results paralleled those in the overall IDA study population (ferumoxytol: $81.1 \%$, vs placebo: $5.5 \%$; $P<0.001$ ). ${ }^{14}$ For the alternative primary end point analysis, mean $\mathrm{Hgb}$ values in ferumoxytol-treated patients increased to $28 \mathrm{~g} / \mathrm{L}$ from baseline to Week 5, compared with a decrease of $1.0 \mathrm{~g} / \mathrm{L}$ for

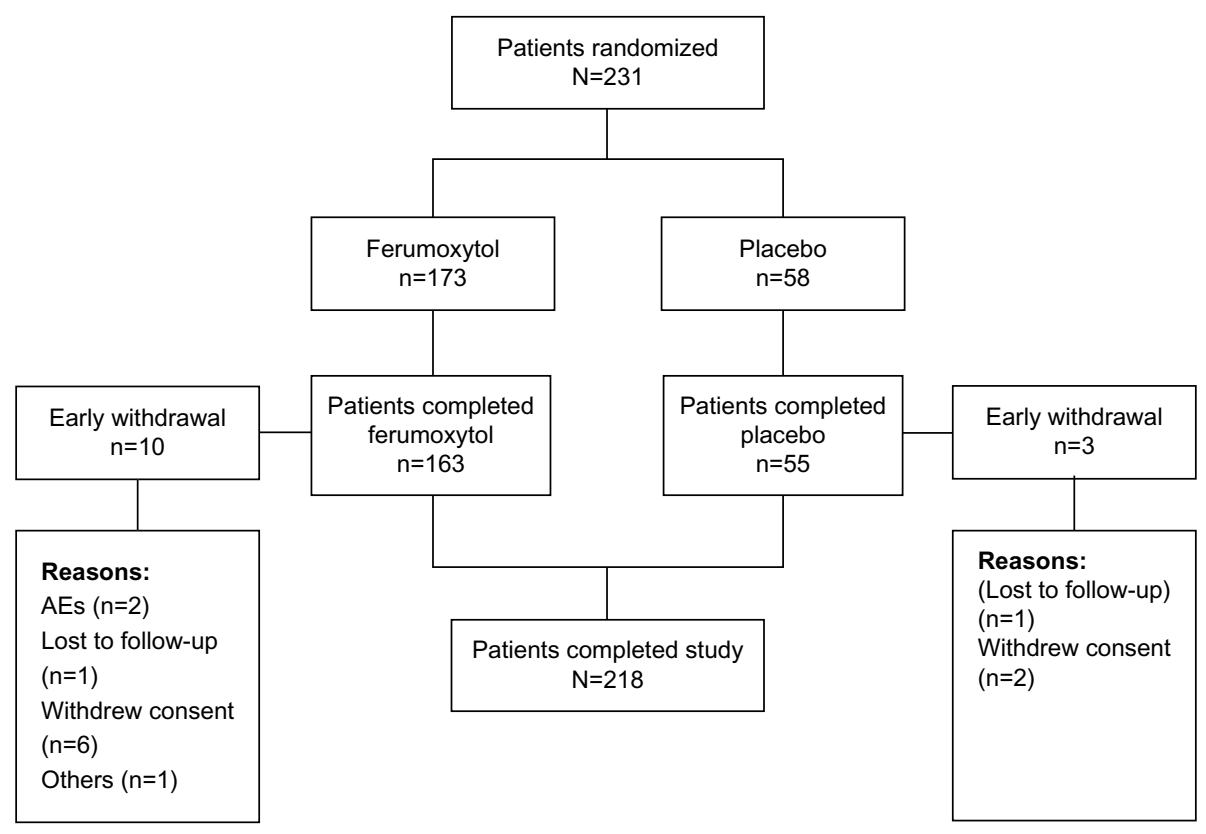

Figure I Patient disposition for the subgroup of patients with IDA and GI disorders. Abbreviations: IDA, iron deficiency anemia; GI, gastrointestinal; $A E$, adverse event. 
Table I Baseline characteristics of the subgroup of patients with IDA and GI disorders (intent-to-treat population)

\begin{tabular}{|c|c|c|c|c|}
\hline \multirow[t]{2}{*}{ Baseline characteristics } & \multicolumn{3}{|c|}{ GI disorders subgroup } & \multirow{2}{*}{$\begin{array}{l}\text { Overall } \\
\text { IDA group } \\
\text { Total } \\
(\mathbf{N}=\mathbf{8 0 8})\end{array}$} \\
\hline & $\begin{array}{l}\text { Ferumoxytol } \\
(n=\mid 73)\end{array}$ & $\begin{array}{l}\text { Placebo } \\
(n=58)\end{array}$ & $\begin{array}{l}\text { Total } \\
(n=23 I)\end{array}$ & \\
\hline \multicolumn{5}{|l|}{ Demographics } \\
\hline Age (years), mean (SD) & $47.4(16.85)$ & $52.1(15.93)$ & $48.6(16.72)$ & $45.1(13.76)$ \\
\hline \multicolumn{5}{|l|}{ Sex, n (\%) } \\
\hline Female & $136(78.6)$ & $44(75.9)$ & I 80 (77.9) & $720(89.1)$ \\
\hline Male & $37(21.4)$ & $14(24.1)$ & $51(22.1)$ & $88(10.9)$ \\
\hline \multicolumn{5}{|l|}{ Race, n (\%) } \\
\hline Asian & $33(19.1)$ & $6(10.3)$ & 39 (16.9) & $130(16.1)$ \\
\hline Black/African-American & $16(9.2)$ & $5(8.6)$ & $21(9.1)$ & $202(25.0)$ \\
\hline White & $120(69.4)$ & $45(77.6)$ & $165(7 \mid .4)$ & $45 \mathrm{I}(55.8)$ \\
\hline Other/multiracial & $4(2.3)$ & $2(3.4)$ & $6(2.6)$ & $25(3.1)$ \\
\hline \multicolumn{5}{|l|}{ Ethnicity, n (\%) } \\
\hline Hispanic/Latino & $19(11.0)$ & $5(8.6)$ & $24(10.4)$ & I 44 (I 7.8$)$ \\
\hline Non-Hispanic/Latino & $154(89.0)$ & $53(91.4)$ & $207(89.6)$ & $664(82.2)$ \\
\hline \multicolumn{5}{|l|}{ Clinical, mean (SD) } \\
\hline Baseline Hgb level ( $g / L)$ & $89(8.9)$ & $87(7.3)$ & $88(8.5)$ & $89(8.9)$ \\
\hline Baseline TSAT (\%) & $6.5(12.97)$ & $4.7(3.53)$ & $6.0(11.67)$ & $6.6(11.51)$ \\
\hline Baseline FACIT-Fatigue & $22.4(11.7)$ & $22.1(11.4)$ & & \\
\hline \multicolumn{5}{|l|}{ Baseline LASA, mean, (SD) } \\
\hline Quality of life & $46.1(21.9)$ & $43.4(23.4)$ & & \\
\hline Activities of daily living & $43.2(22.3)$ & 38.7 (22.9) & & \\
\hline Energy level & $33.7(20.3)$ & $33.2(22.2)$ & & \\
\hline
\end{tabular}

Note: Baseline values were obtained on Day I prior to injection of study drug.

Abbreviations: FACIT-Fatigue, Functional Assessment of Chronic Illness Therapy-Fatigue; GI, gastrointestinal; Hgb, hemoglobin; IDA, iron deficiency anemia; LASA, Linear Analog Scale Assessment; SD, standard deviation; TSAT, transferrin saturation.

patients who received placebo (treatment difference: $29 \mathrm{~g} / \mathrm{L}$; $P<0.001)$. The most rapid increase in mean $\mathrm{Hgb}$ values for ferumoxytol-treated patients occurred between baseline and Week 2, with continued increases through Week 5 (Figure 2). There was little-to-no change in the placebo treatment group over the course of the study.

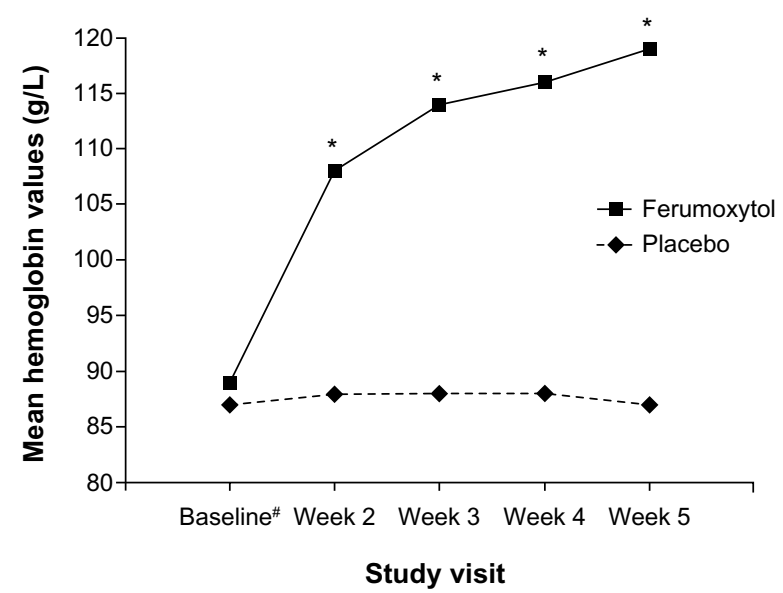

Figure 2 Mean $\mathrm{Hgb}$ values ( $\mathrm{g} / \mathrm{L}$ ) from baseline to Week 5 in patients with IDA and GI disorders (intent-to-treat population).

Notes: "The first ferumoxytol dose was administered at baseline. $* P<0.000 \mathrm{I}$ for mean change from baseline.

Abbreviations: $\mathrm{Hgb}$, hemoglobin; IDA, iron deficiency anemia; Gl, gastrointestinal.
Secondary end points also significantly favored ferumoxytol treatment. Fifty-one percent of ferumoxytol-treated patients achieved a $\mathrm{Hgb}$ level $\geq 120 \mathrm{~g} / \mathrm{L}$ at any time from baseline to Week 5 compared to none of the patients who received placebo (treatment difference: $51.4 \% ; P<0.001$ ). In addition, the mean change in TSAT values from baseline to Week 5 was significantly greater for ferumoxytol-treated patients compared with placebo-treated patients (treatment difference: $11.2 \% ; P<0.001)$, demonstrating the successful repletion of iron stores with ferumoxytol. Mean TSAT values in ferumoxytol-treated patients increased rapidly from baseline through Week 2 and then declined slightly through Week 5 as the Hgb levels increased (Figure 3), likely due to iron utilization for hematopoiesis. There was little-to-no change in TSAT in the placebo treatment group.

\section{PRO analyses}

Baseline mean PRO values were similar between the ferumoxytol and the placebo treatment groups for the FACITFatigue (Table 1), SF-36 domains (Figure 4), and LASA domains (Table 1). As expected for a population of patients with IDA, these baseline values were considerably lower than the preestablished US general population norms of 
Table 2 Efficacy end points in the subgroup of patients with IDA and GI disorders (intent-to-treat population)

\begin{tabular}{|c|c|c|c|}
\hline \multirow[t]{2}{*}{ Efficacy end point } & \multicolumn{2}{|c|}{ Treatment groups } & \multirow[t]{2}{*}{$P$-value } \\
\hline & $\begin{array}{l}\text { Ferumoxytol } \\
(n=173)\end{array}$ & $\begin{array}{l}\text { Placebo } \\
(n=58)\end{array}$ & \\
\hline \multicolumn{4}{|l|}{ Primary end point } \\
\hline $\begin{array}{l}\text { Proportion of patients with } \geq 20 \mathrm{~g} / \mathrm{L} \mathrm{Hgb} \text { increase } \\
\text { at any time from baseline to Week } 5, \mathrm{n}(\%)\end{array}$ & $142(82.1)$ & I (I.7) & $<0.001$ \\
\hline \multicolumn{4}{|l|}{ Alternative primary end point ${ }^{\mathrm{a}}$} \\
\hline$\Delta \mathrm{Hgb}(\mathrm{g} / \mathrm{L})$, mean & 28.0 & -1.0 & $<0.00$ I \\
\hline \multicolumn{4}{|l|}{ Secondary end points } \\
\hline $\begin{array}{l}\text { Proportion of patients with } \mathrm{Hgb} \geq 120 \mathrm{~g} / \mathrm{L} \text { at any } \\
\text { time from baseline to Week } 5, \mathrm{n}(\%)\end{array}$ & $89(51.4)$ & 0 & $<0.001$ \\
\hline$\Delta$ TSAT (\%), mean (SD) & $11.5(\mid 5.01)$ & $0.2(2.27)$ & $<0.00$ I \\
\hline \multicolumn{4}{|l|}{ Patient-reported outcomes, ${ }^{a}$ mean (SD) } \\
\hline$\triangle \mathrm{FACIT}$-Fatigue score & II.I (II.5) & $7.4(8.7)$ & 0.031 \\
\hline$\Delta$ SF-36-Physical functioning score & $4.6(9.5)$ & $0.8(7.8)$ & 0.005 \\
\hline$\Delta$ SF-36-Role-Physical score & $5.6(10.2)$ & $2.6(9.6)$ & 0.045 \\
\hline$\Delta$ SF-36-Bodily pain score & $2.9(9.3)$ & $2.0(11.0)$ & 0.557 \\
\hline$\Delta$ SF-36-General health score & $3.4(8.4)$ & $0.7(6.1)$ & 0.023 \\
\hline$\Delta$ SF-36-Vitality & 9.7 (11.9) & $3.9(8.3)$ & $<0.00$ I \\
\hline$\Delta$ SF-36-Social functioning score & $7.0(12.5)$ & I.I (9.5) & 0.001 \\
\hline$\Delta$ SF-36-Role-Emotional score & $4.7(12.8)$ & $\mathrm{I} .5(\mathrm{I} 2.9)$ & 0.103 \\
\hline$\Delta$ SF-36-Mental health score & $5.8(11.6)$ & $0.6(8.3)$ & $<0.002$ \\
\hline$\Delta$ SF-36-Physical component summary score & $3.7(7.6)$ & $1.7(6.8)$ & 0.071 \\
\hline$\Delta$ SF-36-Mental component summary score & $7.0(12.1)$ & $1.5(9.5)$ & 0.002 \\
\hline$\Delta$ SF-36-6D score & $0.10(0.14)$ & $0.03(0.11)$ & $<0.00$ I \\
\hline$\Delta$ LASA-Energy level score & $16.7(26.2)$ & $9.8(19.0)$ & 0.086 \\
\hline$\Delta$ LASA-Activities of daily living score & $13.4(27.2)$ & $9.8(21.8)$ & 0.385 \\
\hline$\Delta$ LASA-Quality of life score & II.8 (27.7) & $8.2(18.0)$ & 0.386 \\
\hline
\end{tabular}

Note: ${ }^{2}$ Change from baseline to Week 5 unless otherwise stated.

Abbreviations: FACIT-Fatigue, Functional Assessment of Chronic Illness Therapy-Fatigue; Gl, gastrointestinal; Hgb, hemoglobin; IDA, iron deficiency anemia; LASA, Linear Analog Scale Assessment; SD, standard deviation; SF-36, 36-Item Short-Form Health Survey; TSAT, transferrin saturation.

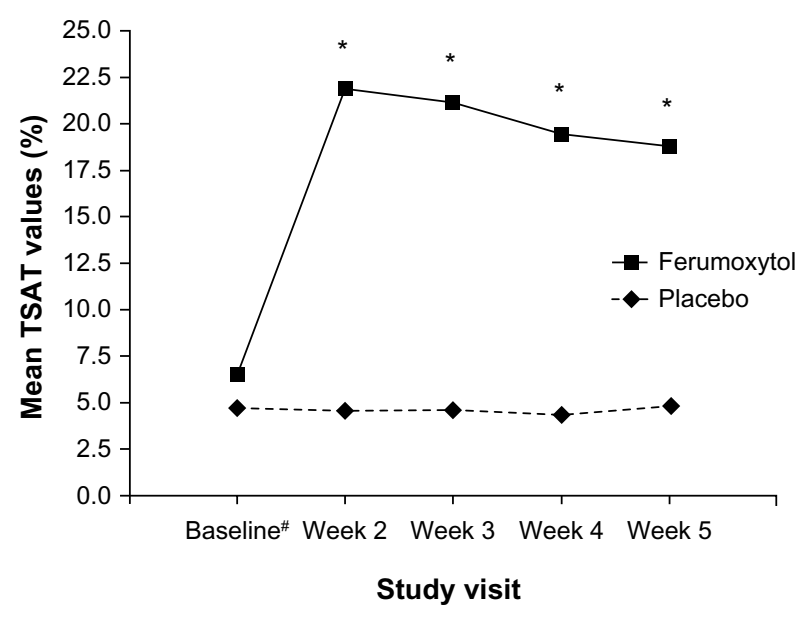

Figure 3 Mean TSAT values from baseline to Week 5 in patients with IDA and GI disorders (intent-to-treat population).

Notes: "The first ferumoxytol dose was administered at baseline. $* P<0.000$ I for mean change from baseline.

Abbreviations: IDA, iron deficiency anemia; GI, gastrointestinal; TSAT, transferrin saturation.
43.6 for FACIT-Fatigue ${ }^{19}$ and 50.0 for SF-36 domain scores (Figure 4) ${ }^{16}$ No population norms are available for the LASA domains.

Ferumoxytol-treated patients demonstrated significantly greater improvements in FACIT-Fatigue and various domains of the SF-36 than placebo-treated patients (Table 2). The mean change in FACIT-Fatigue scores from baseline to Week 5 was 11.1 for the ferumoxytol treatment group compared with 7.4 for the placebo treatment group (least-squares mean [LSM] treatment difference: $3.6 ; P=0.031)$. This treatment difference was greater than the MID (3.0) previously reported for this PRO instrument. Values increased through Week 2 and continued to increase for ferumoxytol-treated patients, while scores decreased after Week 2 in the placebo treatment group (Figure 5). The mean change in SF-36 scores from baseline to Week 5 was significantly higher in the ferumoxytol treatment group compared with the placebo treatment group in six of 


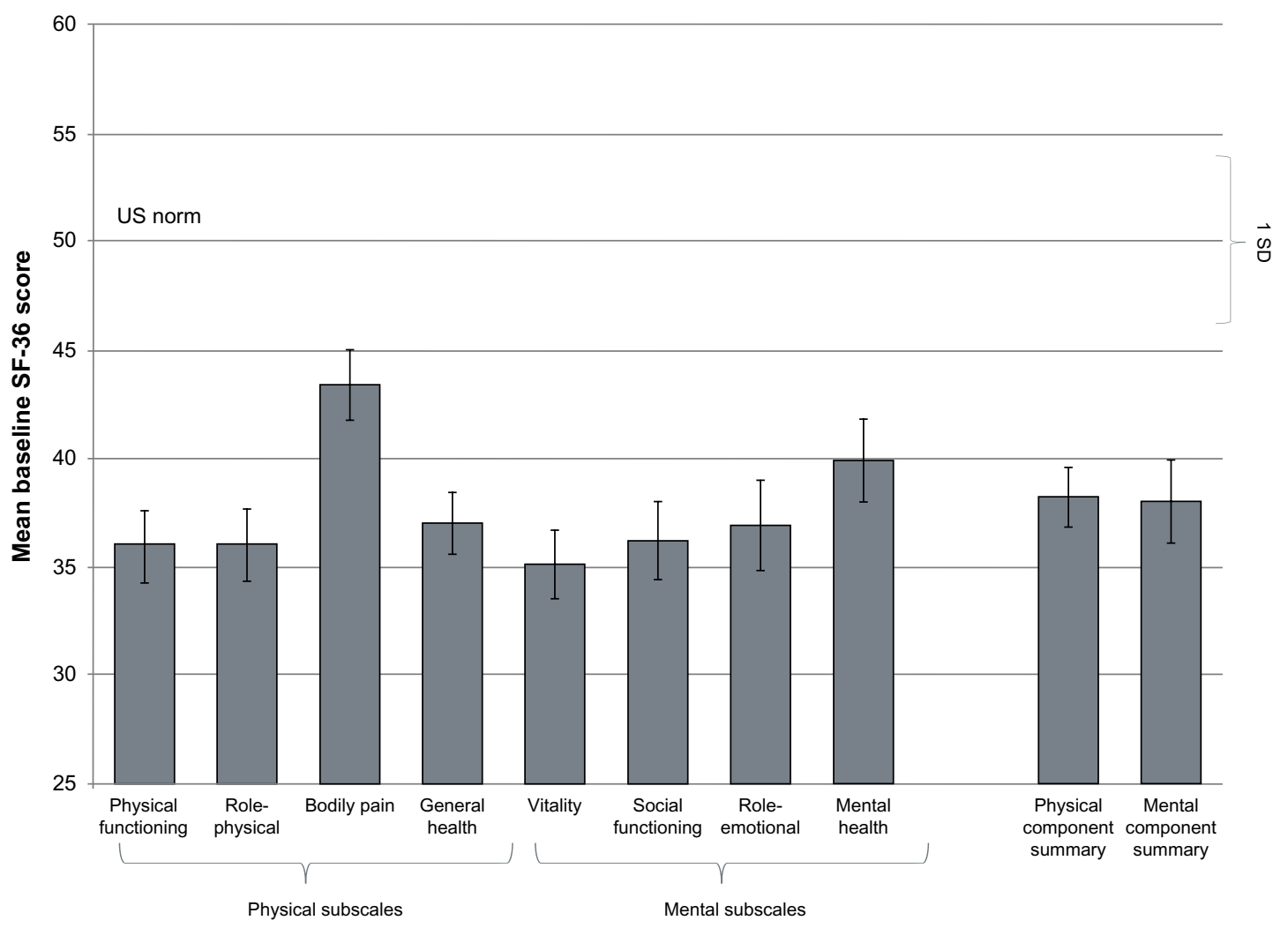

Figure 4 SF-36 domain scores at baseline in patients with IDA and GI disorders (intent-to-treat population).

Abbreviations: IDA, iron deficiency anemia; GI, gastrointestinal; SF-36, 36-item Short-Form Health Survey; SD, standard deviation.

the eight domains assessed (Figure 6). The LSM treatment differences for these domains were physical functioning (3.9; $P=0.005)$, role-physical $(3.1 ; P=0.045)$, general health (2.7; $P=0.023)$, vitality $(5.8 ; P<0.001)$, social functioning

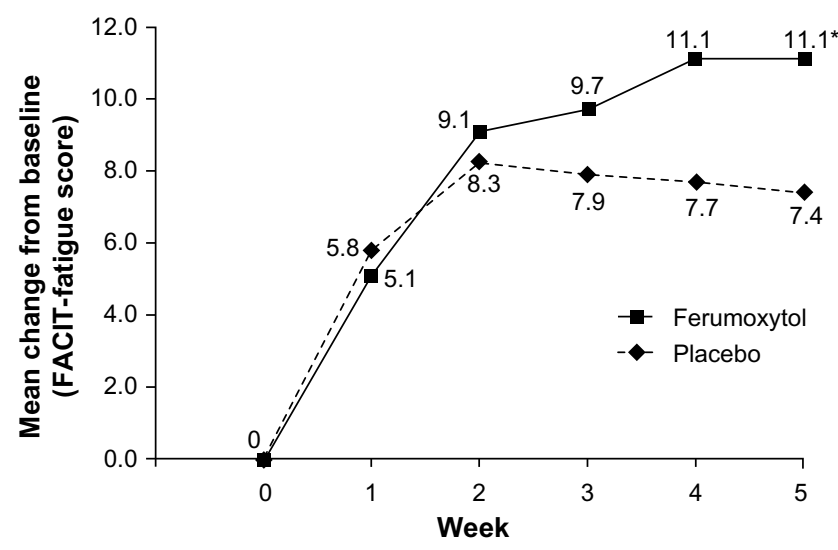

Figure 5 Patient-reported outcomes at baseline and Week 5 in patients with IDA and GI disorders (intent-to-treat population).

Notes: The figure shows mean change from baseline in Functional Assessment of Chronic Illness Therapy (FACIT)-Fatigue scores. $* \mathrm{P}<0.05$ vs placebo. The first ferumoxytol dose was administered at baseline. FACIT-fatigue score is $0-52$, based on 13 questions scored 0-4.

Abbreviations: IDA, iron deficiency anemia; GI, gastrointestinal.
(5.9; $P<0.001)$, and mental health $(5.2 ; P<0.002)$. There were also significantly greater improvements in ferumoxytoltreated patients than in those who received placebo in the MCS (5.5; $P=0.002)$ and in the SF-6D Index scores $(0.07$; $P<0.001)$. The treatment differences between the ferumoxytol and placebo treatment groups in MCS, SF-36-Vitality, and Mental health scores were greater than the previously reported MID for these domains (5.0).

The effect of treatment on LASA-Energy, LASA-Quality of life, and LASA-Activities of daily living scores are summarized in Figure 7. Scores for these domains increased through Week 3 and remained steady until Week 5 in both the ferumoxytol and the placebo treatment groups. Changes from baseline to Week 5 for LASA domain scores were not significantly different between the two treatment groups (Figure 7). However, the mean change in LASA-Energy scores from baseline to Week 5 was 16.7 (95\% confidence interval [CI]: 12.3-21.0) for ferumoxytol-treated patients and 9.8 (95\% CI: 4.6-15.1) for placebo-treated patients (LSM treatment difference: $6.8 ; P=0.086$ ). The lower bound $95 \%$ CI of the mean change score was greater than the previously reported MID (9.61) for the ferumoxytol treatment group, but 


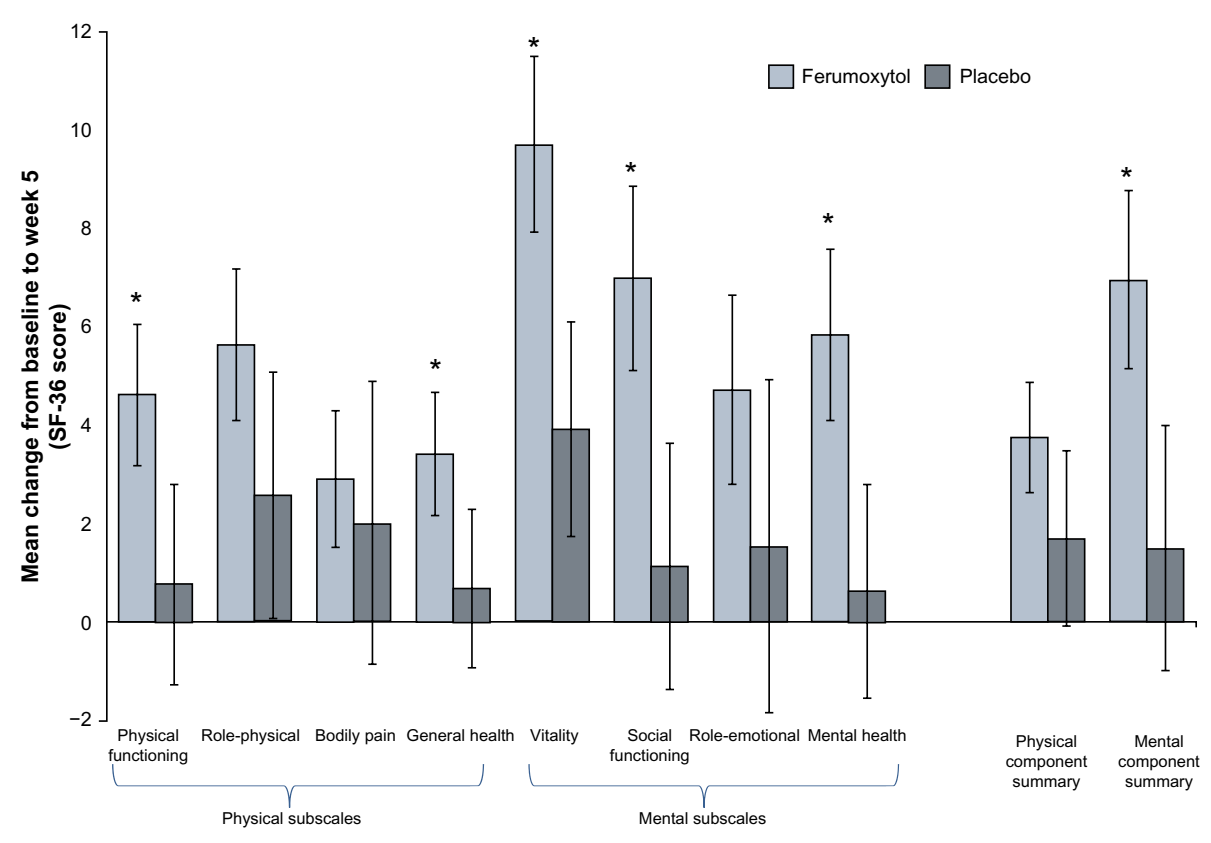

Figure 6 Patient-reported outcomes at baseline and Week 5 in patients with IDA and GI disorders (intent-to-treat population).

Notes: The figure shows mean change from baseline in the 36 -Item Short-Form Health Survey (SF-36) domain scores. $* P<0.05$ vs placebo. The first ferumoxytol dose was administered at baseline. Error bars indicate $95 \%$ confidence intervals.

Abbreviations: IDA, iron deficiency anemia; GI, gastrointestinal.

Table 3 Summary of TEAEs in patients with IDA and GI disorders (safety population)

\begin{tabular}{|c|c|c|c|c|c|c|}
\hline \multirow[t]{3}{*}{ AE category } & \multicolumn{4}{|c|}{ Treatment group } & \multirow{2}{*}{\multicolumn{2}{|c|}{ Total $(\mathbf{N}=231)$}} \\
\hline & \multicolumn{2}{|c|}{ Ferumoxytol $(n=173)$} & \multicolumn{2}{|c|}{ Placebo $(n=58)$} & & \\
\hline & $\begin{array}{l}\text { Events, } \\
\text { n }\end{array}$ & $\begin{array}{l}\text { Patients, } \\
\text { n (\%) }\end{array}$ & $\begin{array}{l}\text { Events, } \\
\text { n }\end{array}$ & $\begin{array}{l}\text { Patients, } \\
\text { n (\%) }\end{array}$ & $\begin{array}{l}\text { Events, } \\
\text { n }\end{array}$ & $\begin{array}{l}\text { Patients, } \\
\text { n (\%) }\end{array}$ \\
\hline All TEAEs & 188 & $84(48.6)$ & 60 & $26(44.8)$ & 248 & $110(47.6)$ \\
\hline Treatment-related AEs & 37 & $20(11.6)$ & 4 & $2(3.4)$ & 41 & $22(9.5)$ \\
\hline SAEs & 6 & $5(2.9)$ & 2 & $2(3.4)$ & 8 & $7(3.0)$ \\
\hline Treatment-related SAEs & 1 & $\mathrm{I}(0.6)$ & 0 & 0 & 1 & $\mathrm{I}(0.4)$ \\
\hline Protocol-defined AEs of special interest ${ }^{\mathrm{a}}$ & 7 & $6(3.5)$ & 1 & I (I.7) & 8 & $7(3.0)$ \\
\hline Composite cardiovascular $\mathrm{AE}$ end point ${ }^{\mathrm{b}}$ & 0 & 0 & 0 & 0 & 0 & 0 \\
\hline AEs resulting in temporary discontinuation of study drug & 0 & 0 & 0 & 0 & 0 & 0 \\
\hline AEs resulting in permanent discontinuation of study drug & 9 & $5(2.9)$ & 0 & 0 & 9 & $5(2.2)$ \\
\hline AEs resulting in study discontinuation & 4 & $2(1.2)$ & 0 & 0 & 4 & $2(0.9)$ \\
\hline Death & 0 & 0 & 0 & 0 & 0 & 0 \\
\hline \multicolumn{7}{|l|}{ TEAEs reported in $\geq 2 \%$ of patients } \\
\hline Headache & 9 & $8(4.6)$ & 3 & $3(5.2)$ & 12 & II (4.8) \\
\hline Nausea & 10 & $9(5.2)$ & 2 & $2(3.4)$ & 12 & II (4.8) \\
\hline Dizziness & 7 & $6(3.5)$ & 2 & $2(3.4)$ & 9 & $8(3.5)$ \\
\hline Diarrhea & 5 & $5(2.9)$ & 2 & $2(3.4)$ & 7 & $7(3.0)$ \\
\hline Urinary tract infection & 5 & $5(2.9)$ & I & $\mathrm{I}(\mathrm{I} .7)$ & 6 & $6(2.6)$ \\
\hline Nasopharyngitis & 5 & $5(2.9)$ & 2 & $2(3.4)$ & 7 & $7(3.0)$ \\
\hline Peripheral edema & 5 & $5(2.9)$ & 1 & $\mathrm{I}(\mathrm{I} .7)$ & 6 & $6(2.6)$ \\
\hline Vomiting & 5 & $5(2.9)$ & 0 & 0 & 5 & $5(2.2)$ \\
\hline Back pain & 4 & $4(2.3)$ & 1 & I (I.7) & 5 & $5(2.2)$ \\
\hline Fatigue & 3 & $3(1.7)$ & 2 & $2(3.4)$ & 5 & $5(2.2)$ \\
\hline Pain in extremity & 4 & $4(2.3)$ & 0 & 0 & 4 & $4(1.7)$ \\
\hline Abdominal pain & 3 & $3(1.7)$ & 3 & $2(3.4)$ & 6 & $5(2.2)$ \\
\hline Dyspnea & 5 & $5(2.9)$ & 1 & I (I.7) & 6 & $6(2.6)$ \\
\hline Anemia & I & I $(0.6)$ & 2 & $2(3.4)$ & 3 & $3(1.3)$ \\
\hline
\end{tabular}

Notes: alncludes moderate-to-severe hypotension requiring medical intervention or hospitalization, acute decreases in systolic blood pressure from baseline of $\geq 30 \%$ during the 60-minute postdose observation period, hypotension associated with symptoms, systemic allergic reactions (anaphylaxis/anaphylactoid reactions), and milder symptoms of hypersensitivity. 'Includes myocardial infarction, heart failure, moderate-to-severe hypertension, and hospitalization due to any cardiovascular cause. Abbreviations: AE, adverse event; GI, gastrointestinal; IDA, iron deficiency anemia; SAE, serious adverse event; TEAE, treatment-emergent adverse event. 

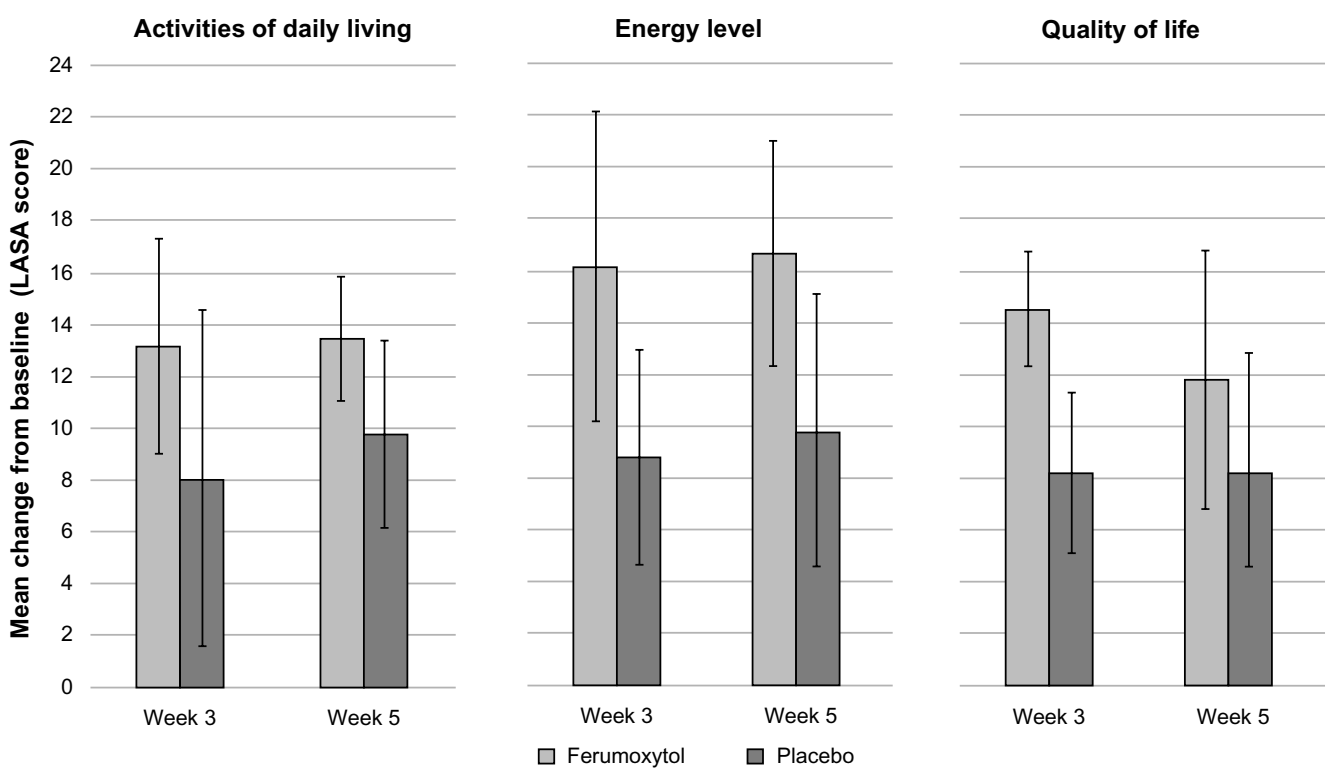

Figure 7 Patient-reported outcomes at baseline, Week 3, and Week 5 in patients with IDA and GI disorders (intent-to-treat population).

Notes: Mean change from baseline in Linear Analog Scale Assessment (LASA) domain scores is shown. The first ferumoxytol dose was administered at baseline. Error bars indicate $95 \%$ confidence intervals.

Abbreviations: IDA, iron deficiency anemia; GI, gastrointestinal.

not for the placebo treatment group. Improvements in these domain scores were also generally similar to those seen in the overall study population. ${ }^{14}$

\section{Safety analyses}

Overall, the incidence of AEs in the subgroup of patients with IDA and underlying GI disorders mirrored those seen in the overall IDA study population in the original Phase III study. The incidence of TEAEs is summarized in Table 3, with no individual TEAE occurring in $>5.2 \%$ of patients in either treatment arm of the GI subgroup. The most common TEAEs occurring in the ferumoxytol treatment group included nausea $(5.2 \%)$, headache $(4.6 \%)$, and dizziness (3.5\%). Overall, TEAEs occurred in more patients in the ferumoxytol treatment group $(11.6 \% ; n=20 / 173)$ compared with the placebo group $(3.4 \% ; n=2 / 58)$. However, the rate of AEs that resulted in discontinuation of study drug was low in both the ferumoxytol and placebo treatment groups $(2.9 \%$ vs $0.0 \%$, respectively).

Rates of SAEs were similar in the ferumoxytol (2.9\%) and placebo (3.4\%) groups, and there was only one treatmentrelated SAE in a ferumoxytol-treated patient. One patient developed symptoms of itching, facial edema, and stridor (without hypotension) within minutes of receiving the first dose of ferumoxytol; the patient responded to treatment (IV steroids and fluids) within minutes, the event resolved, and the patient was sent home the same day. The rate of protocol-defined AESIs (ie, hypotension and hypersensitivity) was higher in ferumoxytol-treated patients $(3.5 \% ; n=6 / 173)$ compared with placebo-treated patients $(1.7 \% ; n=1 / 58)$. There were no cardiovascular composite end point AEs or deaths in either treatment arm of the GI disorders subgroup.

\section{Discussion}

This analysis focused on the subgroup of patients with IDA and an underlying diagnosis of a GI disorder from within a large, randomized, placebo-controlled study of patients with IDA and a history of unsatisfactory oral iron therapy or in whom oral iron could not be used. IV iron was delivered as two IV doses of $510 \mathrm{mg}$ each of ferumoxytol, which was shown to be superior to placebo for all hematologic outcomes, including the primary end point of the proportion of patients achieving a $\geq 20 \mathrm{~g} / \mathrm{L}$ increase in $\mathrm{Hgb}$ at any time from baseline to Week 5. Importantly, the majority of analyzed PRO-related end points also showed statistically significant improvements in ferumoxytol-treated patients compared with those who received placebo. These efficacy results were consistent with the results achieved in the overall Phase III IDA study population. ${ }^{14}$ Also consistent with the overall IDA study population, similar rates of AEs were observed in the ferumoxytol and placebo groups for the majority of AE categories, although treatment-related AEs and AESIs were numerically higher in ferumoxytol-treated patients in both the overall population and in patients with underlying GI disorders. Overall, the incidence of reported AEs in this study was consistent with the safety information 
previously reported from studies of ferumoxytol in patients with CKD..$^{14,21}$

Ferumoxytol is not the only IV iron that has been investigated in the treatment of patients with GI disorders and IDA; however, the magnitude of the observed Hgb increase tended to be higher with ferumoxytol ( $28 \mathrm{~g} / \mathrm{L}$ at Week 5 after administration) than that reported with other IV products administering similar or greater amounts of elemental iron. For example, in a single-arm study, 4 weeks of treatment with low-molecular-weight iron dextran (LMWID) for patients with IBD $(n=50)$ was associated with a mean Hgb increase of $17 \mathrm{~g} / \mathrm{L}$ after the administration of total iron doses ranging from $800 \mathrm{mg}$ to $1,600 \mathrm{mg} .{ }^{22} \mathrm{In}$ another case-matched study in patients with IBD, 8 weeks of treatment with LMWID (mean total iron dose: $949 \mathrm{mg}$ ) was associated with a mean $\mathrm{Hgb}$ increase of $20 \mathrm{~g} / \mathrm{L}$, which was significantly greater than the results from oral iron $(6 \mathrm{~g} / \mathrm{L} ; P<0.01) .{ }^{23}$ The increases in $\mathrm{Hgb}$ in this study with ferumoxytol were accomplished with a treatment duration of 3-8 days compared with a treatment duration of 4-8 weeks in studies with LMWID.

In a randomized controlled trial in patients with IBD, ferric carboxymaltose (median dose: 1,405.5 mg; range: 927-2,102 mg) was found to be noninferior to oral ferrous sulfate (100 mg twice daily for 12 weeks). ${ }^{24} \mathrm{Hgb}$ increased from $87 \mathrm{~g} / \mathrm{L}$ at baseline to $123 \mathrm{~g} / \mathrm{L}$ at the Week 12 end point in the ferric carboxymaltose treatment group (median increase: $37 \mathrm{~g} / \mathrm{L}$; range: $-18 \mathrm{~g} / \mathrm{L}$ to $93 \mathrm{~g} / \mathrm{L}$ ) and from $91 \mathrm{~g} / \mathrm{L}$ to $121 \mathrm{~g} / \mathrm{L}$ in the ferrous sulfate treatment group (median increase: $28 \mathrm{~g} / \mathrm{L}$; range: $-12 \mathrm{~g} / \mathrm{L}$ to $84 \mathrm{~g} / \mathrm{L}$ ); however, the difference between the two groups was not statistically significant $(P=0.70)$.

Similarly, IV iron sucrose has also been studied in patients with IDA and GI disorders. In a randomized 20-week study of patients with IBD $(n=91)$, more patients treated with iron sucrose (mean dose: 1,708 $\mathrm{mg}$ ) achieved a Hgb increase $\geq 20 \mathrm{~g} / \mathrm{L}$ compared with oral iron, but again this difference was not statistically significant ( $66 \%$ vs $47 \%$, respectively; $P=0.07) .{ }^{25}$ By comparison, $82.1 \%$ of patients treated with ferumoxytol in the analysis reported here achieved an $\mathrm{Hgb}$ increase $\geq 20 \mathrm{~g} / \mathrm{L}$ after 5 weeks of follow-up. A recently published Phase III study (NCT01114204) compared ferumoxytol to iron sucrose for the treatment of IDA in adult patients without CKD. ${ }^{21}$ Among the subgroup of 204 patients with an underlying GI disorder in that study, ferumoxytol was shown to be noninferior for the proportion of patients with a $>20 \mathrm{~g} / \mathrm{L}$ increase in $\mathrm{Hgb}$ (ferumoxytol, 80.4\%; iron sucrose, $80.3 \%$, with the lower bound of the $95 \%$ CI [1.5\%] above the predefined noninferiority margin [15\%]) and for the mean change in $\mathrm{Hgb}$ at any time from baseline to Week $5(27 \mathrm{~g} / \mathrm{L}$ increase in Hgb with ferumoxytol vs $25 \mathrm{~g} / \mathrm{L}$ with iron sucrose). ${ }^{21,26}$

Results of this analysis of patients with IDA and GI disorders demonstrated that IDA is associated with very poor PRO scores in patients who had failed oral iron therapy, and that treatment of their IDA is associated with not only an improvement in laboratory parameters but also meaningful changes in most PRO scores. These results were consistent with the results achieved in a full analysis of patients' HRQoL in the overall IDA study population. Analysis of HRQoL outcomes in the overall IDA study population also showed very poor baseline HRQoL scores and treatment with ferumoxytol resulted in clinically meaningful improvements in HRQoL outcomes, across all HRQoL domains, compared with placebo. ${ }^{27}$ Thus, improvements in Hgb status and iron stores appear to be associated with improvements in PROs. The baseline levels of patient-reported fatigue as measured by the FACIT-Fatigue scale were $>15$ points $(\mathrm{SD}=1.5)$ below the population norm, with three points considered to indicate a clinically meaningful decrement. This level of fatigue is in line with that previously reported in anemic cancer patients ( mean $=23.9$; $\mathrm{SD}=12.6$ ).${ }^{19}$ Likewise, the baseline LASA-Energy mean scores were similar to those reported by cancer patients with anemia (mean $=37.2-39.6)^{28}$ and the SF-36-Vitality domain scores were $>10$ points $(\mathrm{SD}=1)$ below the population norm. The superiority of ferumoxytol over placebo for reducing patient fatigue (FACIT-Fatigue) and increasing physical functioning, physical role, general health, vitality, social functioning, and mental health represents a clinically significant benefit for this group of patients who had been previously treated with and failed oral iron treatment. Of importance, the increase in three of the PRO scores (MCS, vitality, and mental health) associated with ferumoxytol treatment in the GI disorders subgroup met or exceeded the established MIDs for those instruments. This suggests that the effect may be greater for mental quality of life than for physical quality of life in this patient population. While the differences between treatment groups in LASA-Energy, -Activity, and -Quality of life scores between ferumoxytoland placebo-treated patients were of similar magnitude to those seen in the overall IDA study population, they were only statistically different for the overall population. ${ }^{27}$

The rates of TEAEs, treatment-related TEAEs, and AESIs (ie, hypotension and hypersensitivity reactions) for ferumoxytol in patients with IDA and GI disorders were similar to those in the overall IDA study population. ${ }^{14}$ Notably, the rates of SAEs were low for both treatment groups in both the overall study population as well as in the GI disorders 
subgroup $(2 \%-3 \%)$. From a safety perspective, these data suggest that the patients with GI disorders tolerated ferumoxytol treatment similarly well to the overall population of patients with IDA. This observation is important as a large proportion of patients with IDA also have underlying GI disorders. ${ }^{1,2}$

These data demonstrate the utility of ferumoxytol as a potential treatment option for the management of IDA in patients with GI disorders who fail treatment with or who cannot tolerate oral iron therapy. Ferumoxytol demonstrated the ability to correct $\mathrm{Hgb}$ levels (defined as an increase in $\mathrm{Hgb}$ of $\geq 20 \mathrm{~g} / \mathrm{L}$ ) in $>80 \%$ of these patients in two treatment visits (two doses of $510 \mathrm{mg}$ ). Furthermore, this analysis found that patients with IDA secondary to GI disorders, who had been unsuccessfully treated with or could not tolerate oral iron, had very poor baseline quality of life scores and that treatment with ferumoxytol, in addition to increasing $\mathrm{Hgb}$, resulted in clinically significant improvements in multiple measures of quality of life for these patients.

A potential limitation of this study is that it is a subgroup analysis from a larger study. ${ }^{14}$ Although this may be considered a weakness of this study, it is important to note that the GI disorders subgroup analysis was prespecified as part of the larger study. A potential strength of this study is the use of placebo-controlled PROs, which is important because of the potential for large placebo response effects in PRO studies.

\section{Conclusion}

In this study, ferumoxytol was shown to be well tolerated and efficacious in the treatment of patients with IDA and underlying GI disorders who had a history of unsatisfactory oral iron therapy or in whom oral iron could not be used. Ferumoxytol may provide an additional option for the treatment of IDA in patients with an underlying GI disorder.

\section{Acknowledgments}

Robert Schupp and Bret Fulton on behalf of inScience Communications, Springer Healthcare (Philadelphia, PA, USA), provided medical writing support funded by AMAG Pharmaceuticals, Inc. Both the primary study and the writing of the secondary analysis were supported financially by AMAG Pharmaceuticals, Inc.

\section{Disclosure}

DCF received payment as an individual for consulting fees or honorarium from AMAG Pharmaceuticals, Inc, in addition to receiving payment for lectures, including service on advisory boards for Takeda Canada Inc. KB, WES, NVD, and ZL are employees of AMAG Pharmaceuticals, Inc, and hold equity in the company. LFA was an employee of AMAG Pharmaceuticals, Inc, at the time of the study and writing of the manuscript. DH received payment from AMAG Pharmaceuticals, Inc, for carrying out separate studies on ferumoxytol. CFB reports no conflicts of interest in this work.

\section{References}

1. Majid S, Salih M, Wasaya R, Jafri W. Predictors of gastrointestinal lesions on endoscopy in iron deficiency anemia without gastrointestinal symptoms. BMC Gastroenterol. 2008;8:52.

2. Niv E, Elis A, Zissin R, Naftali T, Novis B, Lishner M. Iron deficiency anemia in patients without gastrointestinal symptoms - a prospective study. Fam Pract. 2005;22(1):58-61.

3. Carter D, Levi G, Tzur D, Novis B, Avidan B. Prevalence and predictive factors for gastrointestinal pathology in young men evaluated for iron deficiency anemia. Dig Dis Sci. 2013;58(5):1299-1305.

4. Liu K, Kaffes AJ. Iron deficiency anaemia: a review of diagnosis, investigation and management. Eur J Gastroenterol Hepatol. 2012; 24(2):109-116.

5. McCann JC, Ames BN. An overview of evidence for a causal relation between iron deficiency during development and deficits in cognitive or behavioral function. Am J Clin Nutr. 2007;85(4):931-945.

6. Bang SM, Lee JO, Kim YJ, et al. Anemia and activities of daily living in the Korean urban elderly population: results from the Korean Longitudinal Study on Health and Aging (KLoSHA). Ann Hematol. 2013;92(1):59-65.

7. Ando K, Morita S, Higashi T, et al. Health-related quality of life among Japanese women with iron-deficiency anemia. Qual Life Res. 2006;15(10):1559-1563.

8. Barton JC, Barton EH, Bertoli LF, Gothard CH, Sherrer JS. Intravenous iron dextran therapy in patients with iron deficiency and normal renal function who failed to respond to or did not tolerate oral iron supplementation. Am J Med. 2000;109(1):27-32.

9. Kulnigg S, Gasche C. Systematic review: managing anaemia in Crohn's disease. Aliment Pharmacol Ther. 2006;24(11-12):1507-1523.

10. Schroder $\mathrm{O}$, Mickisch $\mathrm{O}$, Seidler $U$, et al. Intravenous iron sucrose versus oral iron supplementation for the treatment of iron deficiency anemia in patients with inflammatory bowel disease-a randomized, controlled, openlabel, multicenter study. Am J Gastroenterol. 2005;100(11):2503-2509.

11. Van Assche G, Dignass A, Bokemeyer B, et al; European Crohn's and Colitis Organisation. Second European evidence-based consensus on the diagnosis and management of ulcerative colitis part 3: special situations. J Crohns Colitis. 2013;7(1):1-33.

12. AMAG Pharmaceuticals, Inc. Feraheme (ferumoxytol) Injection [Prescribing Information]. Waltham, MA: AMAG Pharmaceuticals, Inc; 2015.

13. Jahn MR, Andreasen HB, Futterer S, et al. A comparative study of the physicochemical properties of iron isomaltoside 1000 (Monofer), a new intravenous iron preparation and its clinical implications. Eur J Pharm Biopharm. 2011;78(3):480-491.

14. Vadhan-Raj S, Strauss W, Ford D, et al. Efficacy and safety of IV ferumoxytol for adults with iron deficiency anemia previously unresponsive to or unable to tolerate oral iron. Am J Hematol. 2014;89(1):7-12.

15. Yellen SB, Cella DF, Webster K, Blendowski C, Kaplan E. Measuring fatigue and other anemia-related symptoms with the Functional Assessment of Cancer Therapy (FACT) measurement system. J Pain Symptom Manage. 1997;13(2):63-74.

16. Ware JE Jr, Sherbourne CD. The MOS 36-item short-form health survey (SF-36). I. Conceptual framework and item selection. Med Care. 1992;30(6):473-483.

17. Patrick DL, Gagnon DD, Zagari MJ, Mathijs R, Sweetenham J; Epoetin Alfa Study Group. Assessing the clinical significance of health-related quality of life (HrQOL) improvements in anaemic cancer patients receiving epoetin alfa. Eur J Cancer. 2003;39(3):335-345. 
18. Cella D, Eton DT, Lai JS, Peterman AH, Merkel DE. Combining anchor and distribution-based methods to derive minimal clinically important differences on the Functional Assessment of Cancer Therapy (FACT) anemia and fatigue scales. J Pain Symptom Manage. 2002;24(6):547-561.

19. Cella D, Lai JS, Chang CH, Peterman A, Slavin M. Fatigue in cancer patients compared with fatigue in the general United States population. Cancer. 2002;94(2):528-538.

20. Norman GR, Sloan JA, Wyrwich KW. Interpretation of changes in health-related quality of life: the remarkable universality of half a standard deviation. Med Care. 2003;41(5):582-592.

21. Hetzel D, Strauss W, Bernard K, Li Z, Urboniene A, Allen LF. A phase III, randomized, open-label trial of ferumoxytol compared with iron sucrose for the treatment of iron deficiency anemia in patients with a history of unsatisfactory oral iron therapy. Am J Hematol. 2014;89(6):646-650.

22. Koutroubakis IE, Oustamanolakis P, Karakoidas C, Mantzaris GJ, Kouroumalis EA. Safety and efficacy of total-dose infusion of low molecular weight iron dextran for iron deficiency anemia in patients with inflammatory bowel disease. Dig Dis Sci. 2010;55(8):2327-2331.

23. Khalil A, Goodhand JR, Wahed M, Yeung J, Ali FR, Rampton DS. Efficacy and tolerability of intravenous iron dextran and oral iron in inflammatory bowel disease: a case-matched study in clinical practice. Eur J Gastroenterol Hepatol. 2011;23(11):1029-1035.
24. Kulnigg S, Stoinov S, Simanenkov V, et al. A novel intravenous iron formulation for treatment of anemia in inflammatory bowel disease: the ferric carboxymaltose (FERINJECT) randomized controlled trial. Am J Gastroenterol. 2008;103(5):1182-1192.

25. Lindgren $\mathrm{S}$, Wikman $\mathrm{O}$, Befrits $\mathrm{R}$, et al. Intravenous iron sucrose is superior to oral iron sulphate for correcting anaemia and restoring iron stores in IBD patients: a randomized, controlled, evaluator-blind, multicentre study. Scand J Gastroenterol. 2009;44(7):838-845.

26. Hetzel D, Strauss W, Bernard K, Li J, Allen L. IV iron treatment of iron deficiency anaemia with ferumoxytol in patients with gastrointestinal disorders unable to take oral iron: a randomized controlled trial versus iron sucrose [Abstract P485]. J Crohns Colitis. 2013;7:S204.

27. Strauss W, Dahl NV, Vadhan-Raj S, et al. Effects of IV iron treatment with ferumoxytol on health related quality of life of patients with iron deficiency anemia. J Commun Support Oncol. In Press 2016.

28. Glaspy J, Bukowski R, Steinberg D, Taylor C, Tchekmedyian S, Vadhan-Raj S. Impact of therapy with epoetin alfa on clinical outcomes in patients with nonmyeloid malignancies during cancer chemotherapy in community oncology practice. Procrit Study Group. J Clin Oncol. 1997;15(3):1218-1234.
Clinical and Experimental Gastroenterology

\section{Publish your work in this journal}

Clinical and Experimental Gastroenterology is an international, peerreviewed, open access journal, publishing all aspects of gastroenterology in the clinic and laboratory, including: Pathology, pathophysiology of gastrointestinal disease; Investigation and treatment of gastointestinal disease; Pharmacology of drugs used in the alimentary tract;

\section{Dovepress}

Immunology/genetics/genomics related to gastrointestinal disease. This journal is indexed on CAS. The manuscript management system is completely online and includes a very quick and fair peer-review system. Visit http://www.dovepress.com/testimonials.php to read real quotes from published authors. 